\title{
Reduction of escape cone losses in luminescent solar concentrators with cholesteric mirrors
}

\author{
L.H. Slooff ${ }^{a^{*}}$ A.R. Burgers ${ }^{a}$, M.G. Debije ${ }^{b}$ \\ ${ }^{a}$ ECN Solar Energy, PO Box 1, 1755 ZG Petten, The Netherlands, \\ ${ }^{\mathrm{b}}$ Eindhoven University of Technology, Polymer Technology, $5600 \mathrm{MB}$ Eindhoven, The Netherlands
}

\begin{abstract}
The Luminescent Solar Concentrator (LSC) consists of a transparent polymer plate containing luminescent particles. Solar cells are connected to one or more sides of the polymer plate. Part of the light emitted by the luminescent particles is guided towards the solar cells by total internal reflection.

About $25 \%$ of the dye emission is typically emitted within the optical escape cone of the matrix material and is lost due to emission from the top. We study the application of selectively-reflective cholesteric layers to reduce these losses. We have implemented these mirrors in the ray-tracing model for the LSC. The simulations show that an optimum in performance can be obtained by selecting an appropriate centre wavelength of the cholesteric mirror.

External Quantum Efficiency measurements were performed on LSC devices with a mc-Si, GaAs or InGaP cell and a dichroic mirror. This mirror shows a similar behavior as the cholesteric mirror. The results show that for a $5 \times 5 \mathrm{~cm}^{2} \mathrm{LSC}$ the mirror does improve the EQE in the absorption range of the dye.
\end{abstract}

Keywords: luminescent solar concentrator, fluorescent solar concentrator, ray-tracing, simulations, cholesteric mirror, dichroic mirror

\section{INTRODUCTION}



Fig.1: Schematic 3D view of a luminescent concentrator. AM 1.5 light is incident from the top. The light is absorbed by a luminescent particle. The luminescence from the particle is randomly emitted. Part of the emission falls within the escape cone (determined by the angle (a)) and is leaving the luminescent concentrator at the side (1). The other part of the luminescence is guided to the Si cell by total internal reflection (2). efficiency of $4 \%$ for a stack of two LSCs connected to GaAs cells. Since then, materials research on e.g. dyes, polymers and mirrors has increased the materials database for use in the LSC. Apart form the availability of materials, the
The concept of the luminescent solar concentrator (LSC) (also known as the fluorescent solar concentrator) has been studied since the late 70's. The concentrator usually consists of a flat polymer plate, doped with luminescent dyes or particles and a solar cell attached to one or more sides, see figure 1 . The incoming sunlight is absorbed by the luminescent dye or particles, which subsequently randomly emit light at a larger wavelength. Part of this light is trapped by total internal reflection and will reach the solar cell where it is converted into electricity. Unlike standard solar concentrators, the luminescent concentrator is able to concentrate both direct and diffuse light, which makes tracking of the sun unnecessary. Of all the publication in this field, the extensive work of Zastrow et al [1] is especially interesting, as it is one of the first publications that extensively reports on the many aspects of the luminescent concentrator, like polymer background absorption losses, dye quantum efficiency, and optical quality of the surfaces of the concentrator plate. Their best result was a power conversion 
modeling of the LSC has also improved substantially. Several groups have reported on the modeling of the LSC [2-8]. In principal two different approaches are used, a detailed balance model which is based on the radiative energy transfer between mesh points in the plate, and a ray-tracing model in which every incoming photon is tracked. We have developed a ray-tracing method that does not model individual luminescent particles or dyes, like in the work of Galagher [7] but that applies statistical averaging of the absorption, which reduces the computation time substantially. Previously we have shown that the model is able to predict the experimental results of reflection and transmission measurements, as well as the photovoltaic response from the LSC [9]. Here we report on the application of a selective top mirror for increasing the performance of the LSC.

\section{THE RAY-TRACING MODEL}

Ray-tracing for LSCs uses basic ray-tracing principles. The main extension to the standard ray-tracing model is the handling of the absorption and emission by the luminescent species in the LSC. The 3-D ray-tracing program described here takes these absorption and emission characteristics into account [9].

In ray-tracing a ray represents light of a certain wavelength travelling in a certain direction. A ray that is incident on an LSC can proceed in two ways; it can be reflected, or transmitted. In ray-tracing random numbers are used to determine the fate of the ray in such a situation. As an example, consider the reflection at an interface and assume that the reflection coefficient is $30 \%$. A random number $r$ is drawn from a uniform distribution in the interval $[0,1]$. If $r<0.3$ we assume the ray is reflected, if $r \geq 0.3$ the ray is transmitted.

The situations where the progress of the ray is determined by random numbers are:

- reflection and transmission at interfaces.

- absorption in the polymer slab

- absorption by the luminescent species

- emission by the luminescent species: the luminescent quantum efficiency determines if there is luminescence or not

- wavelengths of emission: the wavelength is selected from the continuous emission spectrum of the dye used, taking into account that the emission wavelength must be larger than the absorption wavelength

- direction of emission: The direction of the emitted ray is selected randomly from the $4 \pi$ solid angle and, hence, independent of the incident direction.

The ray is followed until it is either leaves the system, or is absorbed somewhere in the system and not emitted again. In this way the reflection, absorption and external quantum efficiency (EQE) can be calculated, but also the losses like absorption losses, reflection losses, or loss due to a luminescent quantum efficiency of the dye from less than $100 \%$. Because of the statistical nature of the ray-tracing process, large numbers of rays, typically 20000 per wavelength, must be traced to obtain curves with sufficiently small noise.

\section{RESULTS}

\subsection{State-of-the art device}

All calculations were done for our standard LSC configuration, which consists of a PMMA plate of $5 \times 5 \mathrm{~cm}^{2}$ doped with Lumogen F Red 305 (BASF) and CRS 040 (Radiant Color). On one side of the waveguide a mc-Si solar cell (efficiency $18 \%, \mathrm{ECN}$ baseline) is attached using microscope immersion oil with a refractive index of 1.49. All parameters needed for the ray-tracing calculations have been determined previously $[9,10]$.

The working principle of the LSC is based on total internal reflection (TIR). When light is incident on an interface between a high refractive index medium and a low refractive index medium, it is refracted according to Snell's law:

$n_{1} \sin \theta_{1}=n_{2} \sin \theta_{2}$ 


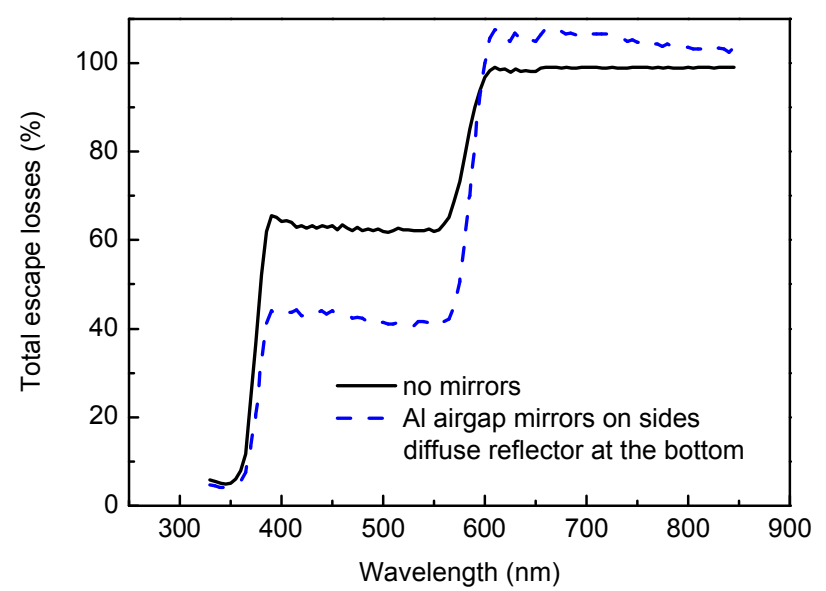

Fig. 2: Total escape losses for an LSC without side mirrors and for an LSC with $85 \%$ aluminum airgap mirrors at the sides and an $85 \%$ diffuse airgap mirror at the bottom. in which $\mathrm{n}_{1}$ is the refractive index of the surrounding media, i.e. air, $\mathrm{n}_{2}$ the refractive index of the LSC plate, $\theta_{1}$ the angle of incidence and $\theta_{2}$ the angle of refraction. From Equation 1 it can be seen that when going from a high refractive index medium to a low refractive index medium, there exists an angle $\left(\theta_{c}\right)$ for which all light is reflected, i.e. it has a reflection coefficient of $100 \%$, or total internal reflection. For the LSC the same holds, but then in 3 dimensions. This leads to the so-called escape cone. Light emitted by a luminescent particle that falls within this escape cone leaves the LSC at the surface, while light that falls outside the escape cone will be subject to TIR. Based on the refractive indices from the polymer plate and air, it can be calculated that if only one solar cell is attached to the plate and the other sides are left uncovered, about $65 \%$ of the emitted light is lost through the surfaces, see Fig. 2. To avoid these losses, mirrors can be applied on the sides. However, by applying mirrors, the refractive index difference between the plate and its surroundings is changed, as air is now replaced by e.g. aluminum. As a consequence of this TIR is lost. In the situation without the mirrors, light that was emitted outside the escape cone was $100 \%$ reflected, but when a mirror is applied it is facing the reflectivity of the aluminum mirror, which is less than $100 \%$. On the other hand, the light that escaped in the situation without the mirrors is now redirected into the plate. To benefit from both TIR and the reflection by the aluminum mirror we simulated an air-gap between the LSC and the mirror. In this way the LSC still has an escape cone, since the surroundings is again air, but the escaping light is reflected by the aluminum mirror. In this way the escape losses in the wavelength region of the dye absorption can be reduced to about $40 \%$ as can be seen in Fig. 2 , where $85 \%$ reflective aluminum side mirrors and an $85 \%$ diffuse reflective bottom mirror was used. The remaining escape losses are mainly due to light escaping from the top surface, where no mirror can be applied. More recently we have applied mirrors with a higher reflectivity of $95 \%$. This resulted in a further increase in performance. The experimental results are given in Table I together with the calculated results from ray-tracing. The more than $100 \%$ escape loss at wavelengths above $600 \mathrm{~nm}$ in Fig. 2 stems from the superposition of light that is entering the plate at that wavelength, is reflected at the back and emitted again, and a contribution form the luminescence of the dye.

Table I: calculated AM 1.5 short circuit current $\left(\mathrm{I}_{\mathrm{sc}}\right)$ and power conversion efficiency, together with the measured efficiency using the ECN solar simulator.

\begin{tabular}{|l|l|l|l|l|l|}
\hline LC plate & calculated Isc & calculated $\eta$ & measured Isc & measured $\eta$ & $\begin{array}{l}\eta \text { bare Si } \\
\text { cell }\end{array}$ \\
\hline $\begin{array}{l}\text { Red } 305+ \\
\text { Flu Yellow }\end{array}$ & $153 \mathrm{~mA}$ & $2.8 \%$ & $147 \mathrm{~mA}$ & $2.7 \%$ & $18.6 \%$ \\
\hline
\end{tabular}

\subsection{Cholesteric top mirror}

From Fig. 2. it can be seen that even with the side and bottom mirror applied, a large fraction of the light escapes from the top. A way to overcome this loss is by application of wavelength selective mirrors. The idea is that the mirror is transparent in the region where the dye is absorbing and highly reflecting in the range where the dye is emitting. The absorption and emission spectra of the dye are shown in Fig. 3, together with the desired transmission and reflection spectrum of the mirror.

One way to realize such a mirror is by applying selectively-reflective chiral nematic (cholesteric) liquid crystal (LC) layer(s) $[11,12]$. In Fig. 4 the optical properties of cholesteric layers are illustrated. 


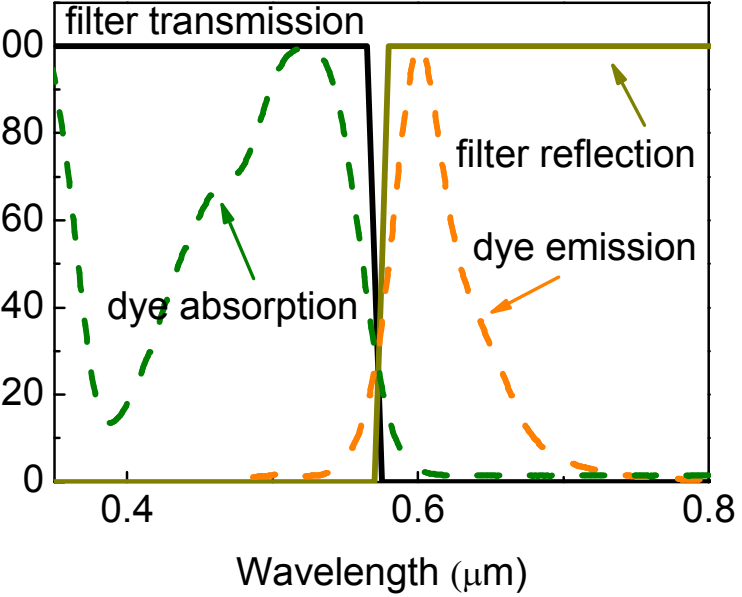

Fig. 3: Absorption and emission spectra of the Red 305 dye, together with the desired transmission and reflection spectrum of the selective mirror.

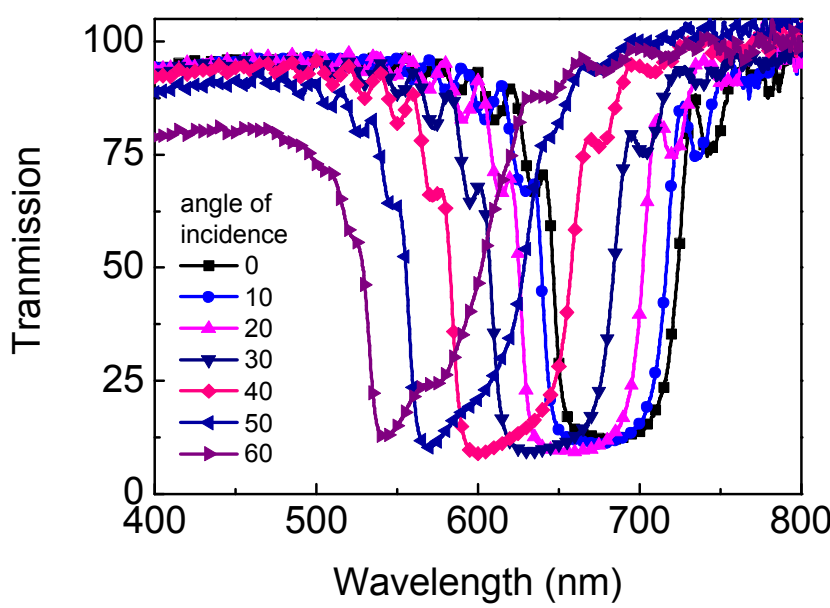

Fig. 4: reflection of a cholesteric layer for different angles of incidence. With increasing angle of incidence the centre wavelength of the high reflection region moves to shorter wavelengths.

As can be seen, the transmission shows the desired low transmission in the dye emission range, but the transmission band is rather small and shows interference fringes. Furthermore, the transmission spectrum depends on the angle of incidence. This will have a large impact on the application of such a mirror in the LSC. The emission by the dye is more or less random and will thus arrive at the top interface under various angles of incidence. So a ray at a wavelength of 600 $\mathrm{nm}$ at normal incidence will not be reflected, as the center wavelength of the cholesteric at this wavelength is $680 \mathrm{~nm}$, but at an angle of $40^{\circ}$ it will be reflected, see Fig. 5. To determine the effect of such a cholesteric mirror, the reflection

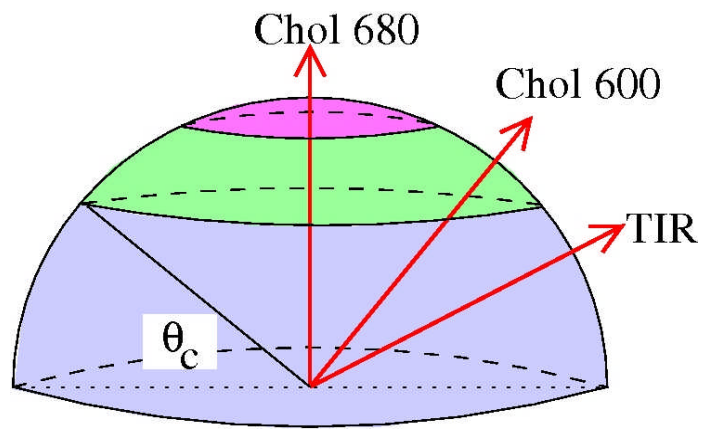

Fig. 5: Schematic view of the different regions the emitted fluorescence of the dye is facing when a cholesteric mirror with a center wavelength of 680 $\mathrm{nm}$ is applied. Light that falls perpendicularly on the top surface faces a reflector with a center wavelength of $680 \mathrm{~nm}$, whereas light that falls in at roughly $45^{\circ}$ faces a reflector with a center wavelength of $600 \mathrm{~nm}$. At even larger angles total internal reflection will take place.

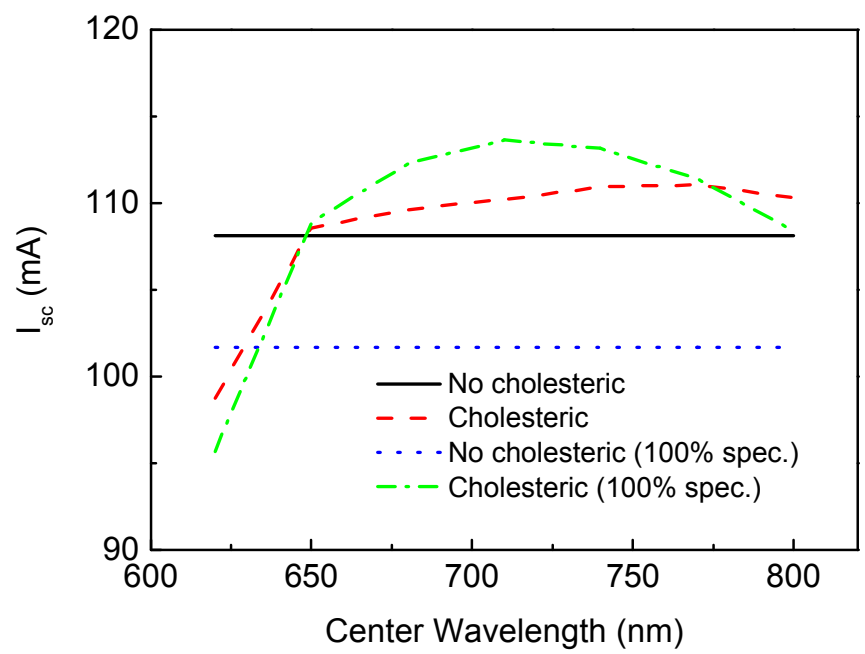

Fig. 6: Calculated short circuit current from the mc-Si cell connected to the LSC plate as a function of the center wavelength of the cholesteric top mirror as compared to the situation where no top mirror is applied. Calculations are shown for a diffuse air-gap bottom mirror, as well as a direct specular bottom mirror $(100 \% \mathrm{spec})$. 

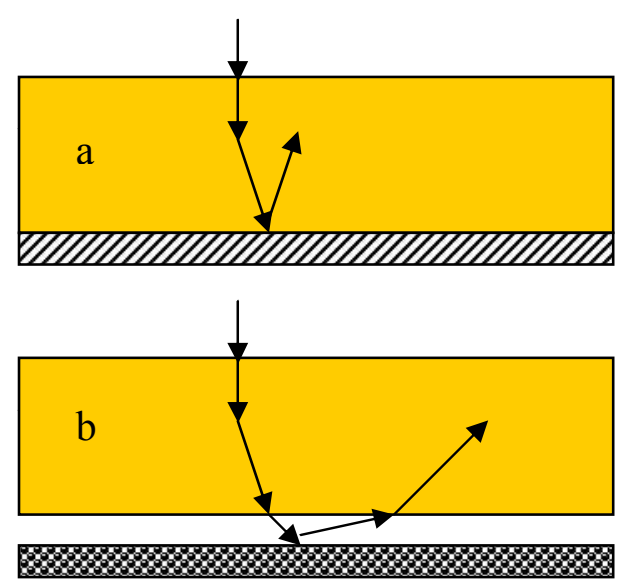

Fig. 7 Schematic picture of the two bottom mirror configurations: a) direct $100 \%$ specular mirror and b) diffuse air-gap mirror

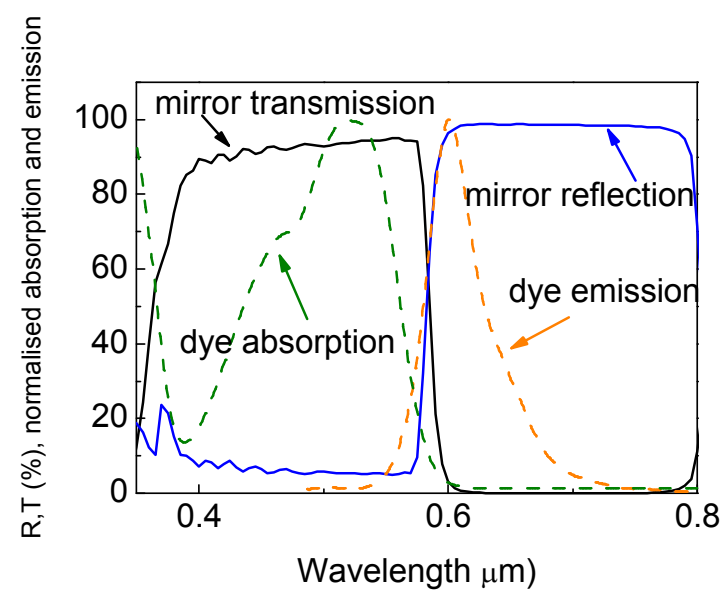

Fig. 8: Absorption and emission spectra of the Red 305 dye, together with the transmission and reflection spectrum of the LOT-ORIEL mirror.

and transmission characteristics of Fig. 4 were implemented in the ray-tracing program [11]. Calculations were performed for an LSC with and without a cholesteric mirror. Fig. 6 shows the current from the attached solar cell, as calculated with the ray-tracing program for different center wavelengths of the cholesteric mirror. The calculations were done for the configuration with a diffuse air-gap mirror at the bottom and for a direct $100 \%$ reflecting specular mirror at the bottom. From this figure it is clear that the center wavelength of the cholesteric mirror needs to be red-shifted substantially for optimal performance. Although the peak emission wavelength of the dye is about $600 \mathrm{~nm}$, the optimal center wavelength of the cholesterics for normal incidence is $710 \mathrm{~nm}$. Furthermore, the effect of the cholesteric mirror is much larger, $12 \%$, in the case of a direct $100 \%$ reflecting specular mirror than for the diffuse air-gap bottom mirror, $3 \%$. The reason for this must be found in the different angular dependence of the reflected rays. Fig. 7 shows a schematic picture of the two configurations. In the case of a diffuse air-gap mirror, the randomly emitted luminescence is scattered at the backside mirror and a fraction of this scattered light will be transported directly to the solar cell, without reaching the top surface. This fraction will not benefit from the application of a cholesteric top mirror. With a direct specular mirror, the randomly emitted luminescence will be specularly reflected and will thus remain randomly oriented. As a result, part of the emission will reflect at the backside and escape from the top side, if emitted within the escape cone. For this reason, the performance of a direct specular bottom mirror is lower than for a diffuse air-gap mirror. However, by applying a cholesteric mirror, the escaping light will be reflected and the light can reach the solar cell.

\subsection{Dichroic mirror}

Unfortunately, it was not possible to immediately verify the ray-tracing results with experiments, due to challenges in the fabrication of the cholesteric mirrors. However, we did find a mirror (590FD24-50SX, LOT-ORIEL) with the desired characteristics. The reflection and transmission spectrum of this mirror is shown in Fig. 8. We used this mirror in combination with an LSC for different types of attached solar cells. The results of external quantum efficiency (EQE) measurements are shown in Fig. 9. For all solar cells used, i.e. mc-Si, GaAs, and InGaP, the EQE shows an improvement in the dye absorption range when the mirror is applied (note that the lower EQE for the InGaP cell is due to a bad electrical contact of the cell). However, the EQE spectrum without filter is somewhat broader, indicating that the cut-off wavelength of the mirror is too blue-shifted, contrary to what could be expected based on the spectra in Fig. 8, where it seems to be slightly too red. The reason is that this dichroic mirror, similar to the cholesteric mirror, shows an angle of incidence dependent reflection and transmission spectrum. So similar to the case of the cholesteric mirror, the cut-off wavelength of the mirror must be red shifted for optimal performance.

The shoulder at wavelengths above $600 \mathrm{~nm}$ in Fig. 9 for the case without the mirror are resulting from light that enters the plate and that is not absorbed by the dye. This light is scattered at the diffuse rear-side mirror and part of it will reach the solar cell, before it leaves the LSC at the top. When the mirror is applied, light incident on the LSC at these wavelengths will be reflected at the top and will thus not enter the plate. As a result, this shoulder disappears in the EQE spectra with the mirror. Above $800 \mathrm{~nm}$ the filter becomes partly transparent again and a small EQE response can be 


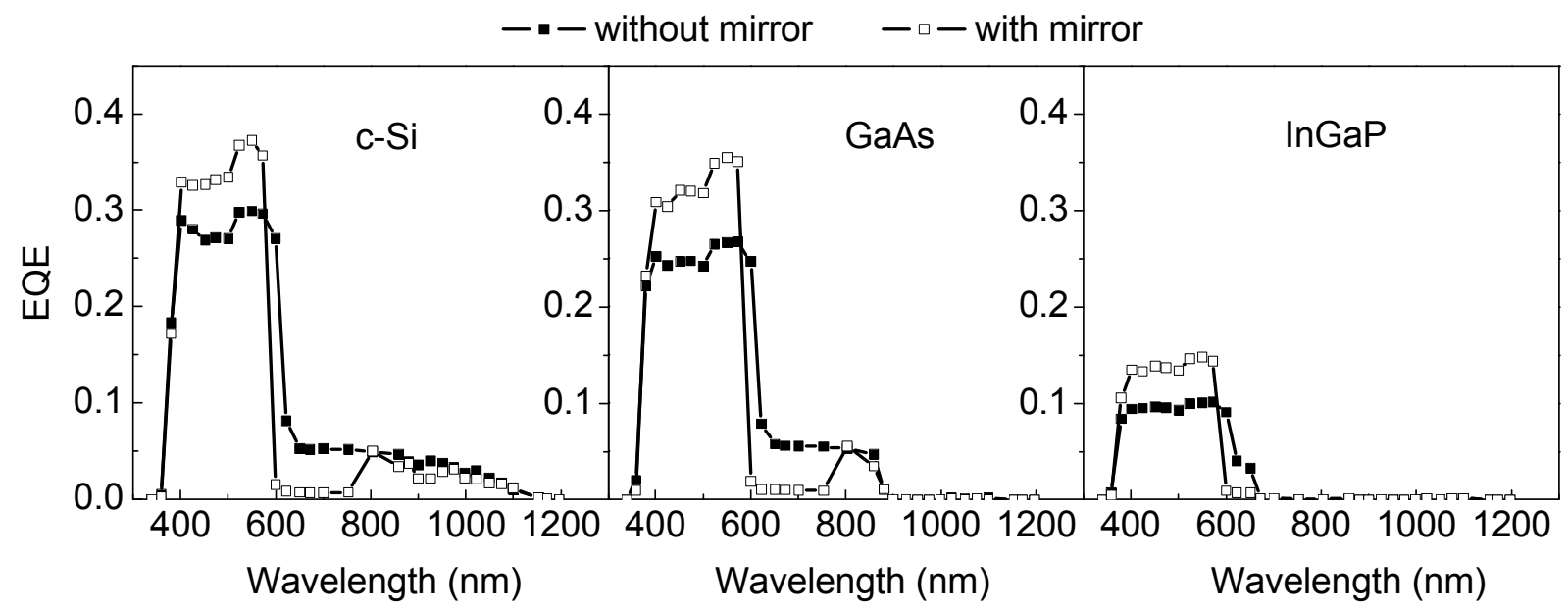

Fig. 9: Absorption and emission spectra of the Red 305 dye, together with the desired transmission and reflection spectrum of the selective mirror.

observed. The relative contribution of the diffusely scattered light compared to the dye contribution is rather large for the LSC with a mc-Si cell, but decreases for the GaAs and InGaP cells due to the wider bandgap of these materials. The contribution will also decrease if larger LSC plates will be used. It can thus be concluded that the dichroic mirror will only result in an increased performance for large, $>25 \mathrm{~cm}^{2}$, LSC plates and for smaller LSC plates only when an InGaP cell is connected.

\section{CONCLUSIONS}

We have shown experimental results on an LSC with specular air-gap mirrors and a diffuse air-gap bottom mirror. The power conversion efficiency of the LSC with a mc-Si solar cell on one side, was $2.8 \%$. To improve the performance we have performed ray-tracing calculations for an LSC with a selective top mirror. For these calculations the optical properties of cholesteric mirrors were used. The result shows an improvement in short circuit current of 3\% for an LSC with a diffuse air-gap bottom mirror and $12 \%$ for an $\mathrm{LC}$ with a direct specular mirror. The effect of a selective top mirror was also shown experimentally by application of a dichroic mirror to the LSC. The improvement in external efficiency ranged from about $20-30 \%$ depending on the type of solar cell that was applied. Due to the influence of diffusely scattered light at the backside mirror of these relatively small LSCs, the overall performance was not improved. It is expected that an overall positive effect of the selective mirror will be obtained for larger LSCs.

\section{ACKNOWLEDGMENTS}

Andreas Büchtemann from the Fraunhofer institute for Applied Polymer research (FhG-IAP) is greatly acknowledged for fabrication of the LSC plates that were used to test our ray-tracing model. This work has been supported in part by European Commission as part of the Framework 6 integrated project FULLSPECTRUM (contract SES6-CT-2003502620).

\section{REFERENCES}

[1] A. Zastrow, thesis, Freiburg (1981)

[2] A.J. Chatten, D.J. Farrell, C.M. Jermyn, P.A. Thomas, B.F. Buxton, A. Büchtemann, R. Danz and K.W.J. Barnham, "Thermodynamic Modelling of the Luminescent Solar Concentrator ", Proc. 31st IEEE Photovoltaic Specialists Conference, Orlando, 82-85 (2005) 
[3] A. J. Chatten, K. W. J. Barnham, B. F. Buxton, N. J. Ekins-Daukes, and M. A. Malik, "Quantum Dot Solar Concentrators ", Semicon., 38, 909-917 (2004)

[4] A.A. Earp, G.B. Smith, P.D. Swift, J. Franklin, "Maximising the light output of a Luminescent Solar Concentrator", Solar Energy 76, 655-667 (2004)

[5] T. Markvart, L. Danos, P. Kittidachachan and R. Greef, "Detailed balance efficiency of ideal single-stage fluorescent collectors", Proceedings of the 20th European Photovoltaic Solar Energy Conference, 171-174 (2005)

[6] M. Carrascosa, S. Unamuno, F. Agullo-Lopez, "Monte Carlo simulation of the performance of PMMA luminescent solar collectors" Appl. Opt. 22, 3236-3241 (1983)

[7] S. J. Gallagher, P. C. Eames, and B. Norton, "Quantum dot solar concentrator behaviour, predicted using a ray trace approach", J. of Ambient Energy 25, 47-56 (2004)

[8] U. Rau, F. Einsele, G.C. Glaeser, "Efficiency limits of photovoltaic fluorescent collectors", Appl. Phys. Lett. 87, 171101 1-171101_3(2005)

[9] A.R. Burgers, L.H. Slooff, R. Kinderman, J.A.M. van Roosmalen, "Modelling of luminescent concentrators by raytracing", Proceedings of the 20th European Photovoltaic Solar Energy Conference, 394-397 (2005)

[10] A.R. Burgers, L.H. Slooff, M.G. Debije "Reduction of escape cone losses in luminescent concentrators with cholesteric mirrors", PVSEC-17 Technical Digest., (2007)

[11] M. G. Debije, R. H. L. van der Blom, D. J. Broer, C. W. M. Bastiaansen, "Using selectivelyreflecting organic mirrors to improve light output from a luminescent solar concentrator", Proceedings of the World Renewable Energy Congress IX, (2006).

[12 ] M. G. Debije, C. W. M. Bastiaansen, D. J. Broer, M.J Escuti, C. Sanchez, Patent WO 2006/088369 A2:

"Luminescent object and utilisation thereof", (2006) 